\title{
ADENOMA HIPOFISÁRIO: CORRELAÇÃO CLÍNICA, LABORATORIAL E RADIOLÓGICA
}

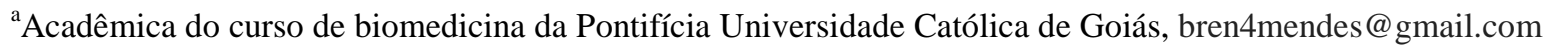

bAcadêmica do curso de biomedicina da Pontifícia Universidade Católica de Goiás, lorenagomesb@yahoo.com.br

${ }^{c}$ Mestre e doutoranda em Biologia Celular e Molecular pelo laboratório de Radiobiologia e Mutagênese da Universidade Federal de Goiás. Docente no curso de Biomedicina da Pontifícia Universidade Católica de Goiás, profacristiene@gmail.com
\end{abstract}

Recebido em: 17/12/2014 - Aprovado em: 04/06/2015 - Disponibilizado em: 15/07/2015

\begin{abstract}
Resumo: Adenomas hipofisários $(\mathrm{AH})$ geralmente são tumores benignos, com sintomas de hiposecreção ou hipersecreção hormonal e/ou relacionados à compressão de tecidos vizinhos. Tais sintomas podem causar complicações que comprometem a qualidade de vida dos pacientes. Entre as massas selares, os AH são a patologia mais comum, todavia, sua distinção com outras patologias não é simples, visto que muitas delas podem ser semelhantes do ponto de vista clínico, endócrino e radiológico. O diagnóstico inicial dos $\mathrm{AH}$ é realizado de acordo com os efeitos provocados pelo crescimento do tumor devido à compressão de estruturas vizinhas e/ou às síndromes de hiperprodução ou hipoprodução hormonal. Os exames laboratoriais usados na dosagem dos hormônios hipofisários e os exames de imagem são essenciais para a elucidação da suspeita clínica de AH. Sendo assim, o objetivo do presente trabalho é informar quanto à necessidade de se estabelecer um diagnóstico precoce e específico para os AH, apontando suas manifestações clínicas, as doenças causadas pelos mesmos e a correlação dos dados clínicos e laboratoriais com os exames radiológicos.
\end{abstract}

Palavras-chave: Adenoma Hipofisário. Alterações Hormonais. Exames de imagem.

\begin{abstract}
Pituitary Adenomas (PA) usually are benign tumors that present symptoms of hypo or hyper hormonal secretion. The symptoms of PA can also be related to compression of nearby tissues that can affect the quality of life of patients. Among the sellar masses, the PA are the most common pathology, however, its differentiation from other pathologies is not simple, because theses pathologies may be similar when related to the clinical, endocrine and radiologic aspects. The initial diagnosis of the PA is performed according to the effects caused by tumor growth, which is due to the compression of adjacent structures and/or syndromes of hypo or hyper hormonal production. Additionally, laboratory exams used for dosage of pituitary hormones and imaging tests are essential for the elucidation of the clinical suspicion of PA. Thus, the objective of this study is to inform the need to establish an early and specific diagnosis for PA, indicating its clinical manifestations, the diseases caused and the correlation of clinical and laboratory data with the radiological examinations.
\end{abstract}

Keywords: Pituitary Adenoma. Hormonal Changes. Image exams. 


\section{INTRODUÇÃO}

Adenomas hipofisários (AH) são tumores caracterizados pelo aumento proliferativo de células adenohipofisárias produtoras de hormônios tróficos, sendo que esses podem ser: o hormônio de crescimento $(\mathrm{GH})$, a corticotropina $(\mathrm{ACTH}), \quad \mathrm{o}$ hormônio tireoestimulante (TSH), o hormônio luteinizante (LH), o hormônio folículoestimulante (FSH), e a prolactina (PRL) (MOLITCH, 2014; LAKE et al., 2013).

Tais tumores geralmente são benignos e permanecem confinados na sela túrcica, entretanto, podem ser invasivos, exibir crescimento acelerado e comprometer tecidos adjacentes (VILAR, 2013).

A prevalência dos $\mathrm{AH}$ é estimada em $16.7 \%$ na população em geral, sendo a patologia mais comum entre as massas selares, respondendo por aproximadamente 90\% dos casos. Além disso, são responsáveis por $10 \%$ dos tumores cerebrais (LAWS et al., 1982 apud CURY et al., 2009; EZZAT, 2004 apud MILANO, 2010).

$\mathrm{O}$ diagnóstico inicial dos $\mathrm{AH}$ é realizado de acordo com os efeitos provocados pelo crescimento do tumor devido à compressão de estruturas vizinhas, tais como, alterações visuais (compressão do nervo óptico) e cefaleia (compressão da duramáter) e/ou às síndromes de hiperprodução (Acromegalia, Doença de Cushing, hiperprolactinemia), ou deficiência da secreção dos hormônios hipofisários (hipopituitarismo) (MELMED \& JAMESON, 2013).

Os exames laboratoriais usados na dosagem dos hormônios hipofisários e os exames de imagem, especialmente a Tomografia Computadorizada (TC) e a Ressonância Magnética (RM), são essenciais para a elucidação da suspeita clínica de adenomas hipofisários.

O objetivo do presente trabalho é informar quanto à necessidade e importância de se estabelecer um diagnóstico precoce e específico para os adenomas hipofisários, apontando suas manifestações clínicas, as doenças causadas pelos mesmos e a correlação dos dados clínicos e laboratoriais com os exames de imagem, visto que o amplo espectro de sintomas endócrinos podem ser confundidos com outras patologias.

\section{METODOLOGIA}

A revisão de literatura foi realizada tendo como base artigos científicos, livros de clínica médica, dissertações e teses que abordaram assuntos relacionados a adenomas hipofisários, com foco nos aspectos clínicos, laboratoriais e radiológicos. As bases eletrônicas consultadas foram Medical Literature Analysis and Retrievel System Online (MEDLINE/PUBMED), Scientific 
Eletronic Library Online (SCIELO) e Literatura Latino-Americana e do Caribe em Ciências da Saúde (LILACS).

Trabalhos de língua portuguesa, inglesa, espanhola e francesa, completos, que atenderam aos critérios de inclusão, publicados entre 2008 e 2014, foram selecionados para o presente estudo. As palavras chave utilizadas foram adenoma hipofisário, alterações hormonais em tumores pituitários, ressonância magnética, tomografia computadorizada, pituitary adenomas, laboratory diagnosis of pituitary tumors, computed tomography and magnetic resonance of the sella turcica.

\section{CLASSIFICAÇÃO GERAL DOS} ADENOMAS

Os tumores de hipófise podem ser classificados de maneira diversificada e complementar, de acordo com alguns critérios, tais como: produção hormonal; célula de origem da expansão clonal; características anátomo-radiológicas; imunohistoquímica e padrões moleculares (DELELLIS et al., 2004 apud GOMES, 2011).

A classificação funcional dos adenomas está relacionada com a produção hormonal, podendo os tumores serem classificados como clinicamente funcionantes (hormonalmente ativos) e clinicamente não funcionantes (hormonalmente inativos).
Entretanto, dentro do grupo clinicamente não funcionante pode haver uma secreção hormonal reduzida ou uma secreção de peptídeos biologicamente inativos. Aproximadamente 2/3 dos tumores diagnosticados são funcionantes (MILKER, 2001 apud CURY et al., 2009).

As classificações anátomoradiológicas baseiam-se na dimensão e no grau de invasão do tumor. Classificam-se em microadenomas ( $\leq 10 \mathrm{~mm}$ de diâmetro) e macroadenomas (>10mm de diâmetro) (HARDY, 1969 apud GOMES, 2011). A classificação pela imunohistoquímica identifica os hormônios produzidos no citoplasma das células tumorais e esclarece a atividade funcional da maioria dos adenomas (DELELLIS et al., 2004 apud GOMES, 2011).

Estudos de biologia molecular por diferenciação de células da adenohipófise demonstram que tais células são determinadas por um padrão de expressão de fatores de transcrição, como também análises ultraestruturais que permitem a observação de grânulos secretórios característicos para cada tipo de hormônio, o que tem permitido complementar o diagnóstico na maioria dos casos de AH (DELELLIS et al., 2004 apud GOMES, 2011; PLATA et al., 2012).

Segundo a classificação da Organização Mundial de Saúde (OMS) existem três tipos de tumores de hipófise: adenomas típicos, atípicos e carcinomas. Os 
adenomas típicos podem ser bem delimitados ou invasivos. Classificam-se como adenomas atípicos os que têm crescimento invasivo para as estruturas periselares e índice mitótico elevado. Consideram-se invasivos quando crescem para qualquer uma das estruturas anatômicas vizinhas, ocorrendo em cerca de 50\% dos casos (SAEGER, 2007 apud ALVES et al., 2011).

Para classificar um tumor hipofisário em carcinoma é necessária a presença de metástases em órgãos distantes ou sistema nervoso central, sem continuidade com o tumor. Os carcinomas da hipófise são raros e representam 0,2\% dos tumores hipofisários (KALTSAS et al., 2005; LOPES, 2005 apud ALVES, 2011).

\section{Adenomas funcionantes e não funcionantes}

Os adenomas funcionantes provocam a secreção exacerbada de hormônios, tais como: $\quad$ Prolactinomas $\quad(\uparrow P R L)$, Somatotropinoma $(\uparrow \mathrm{GH})$, Corticotropinoma ( $\uparrow \mathrm{ACTH}), \quad$ Tireotropinoma $\quad(\uparrow \mathrm{TSH})$, Gonadotropinoma ( $\uparrow \mathrm{LH}, \uparrow \mathrm{FSH})$ ou podem co-secretar dois ou mais hormônios. A incidência dos adenomas funcionantes é em torno de $75 \%$ dos casos, sendo o mais frequente o prolactinoma, seguido dos secretores de GH, GH e PRL, ACTH, TSH, LH e FSH e plurihormonais (LAKE et al., 2013).
AH clinicamente não funcionantes são silenciosos, visto que não produzem clínica de hipersecreção hormonal. Por esse motivo, seus sinais e sintomas dependem de seu efeito expansivo no sistema nervoso central com compressão de estruturas vizinhas e/ou hipopituitarismo. De acordo com Kovacs e cols. (1996 apud MELMED \& KLEINBERG, 2008), devido à falta de sinais e sintomas de hipersecreção endócrinas, o diagnóstico muitas vezes é tardio. No entanto, com o surgimento da ressonância magnética, um número crescente de pacientes foram incidentalmente diagnosticados (MOLITCH, 2008; CURY et al., 2009).

Apesar desses adenomas não produzirem clínica de hipersecreção hormonal, $86 \%$ deles apresentam imunohistoquímica positiva para pelo menos uma subunidade de hormônio glicoprotéico, como por exemplo para FSH, LH ou subunidade- $\alpha$, sendo que $8,1 \%$ são adenomas corticotróficos silenciosos e $2,7 \%$ são adenomas somatotróficos silenciosos (CURY et al., 2009).

\section{ASPECTOS CLÍNICOS DOS ADENOMAS HIPOFISÁRIOS}

\section{Prolactinomas}

A principal causa de hiperprolactinemia patológica são os prolactinomas, que representam $40 \%$ a $60 \%$ 
dos casos de AH funcionante. Com predominância em mulheres - 89\% dos casos descritos na literatura - ocorre em qualquer faixa etária. As manifestações clínicas na mulher são galactorréia, amenorréia, infertilidade, diminuição da libido, dispareunia, osteoporose, acne/hirsutismo e ganho de peso. No homem os prolactinomas são, na sua maioria, macroadenomas, e podem provocar diminuição da libido, infertilidade, ginecomastia, disfunção erétil, osteoporose, ganho de peso e galactorréia (VELOZA \& PRAZERES, 2011; VILAR, 2013).

\section{Corticotropinoma}

Os corticotropinomas, em geral microadenomas, representam aproximadamente $90 \%$ dos casos de Síndrome de Cushing (SC) endógena em adultos. O diagnóstico é realizado em duas etapas: inicialmente por meio do diagnóstico da SC, posteriormente pelo diagnóstico etiológico propriamente dito (MELMED \& JAMESON, 2013).

O diagnóstico sindrômico é realizado por meio da presença de manifestações clínicas sugestivas de hipercortisolismo associadas a exames laboratoriais confirmatórios da hipersecreção de cortisol. As manifestações clínicas mais específicas da SC incluem: obesidade ou ganho ponderal, fragilidade capilar, face de lua cheia, estrias cutâneas violáceas, hirsutismo, pletora, fraqueza muscular proximal e osteopenia/osteoporose. Em crianças, a característica típica é o ganho de peso com redução da velocidade de crescimento (MELMED \& JAMESON, 2013).

Entre as manifestações menos específicas, estão a obesidade centrípeta, a hipertensão arterial sistêmica, hiperglicemia, transtornos psiquiátricos e o hipogonadismo/irregularidade menstrual. Uma situação clínica que deve sempre exigir atenção médica é a utilização de glicocorticóides exógenos, que podem corresponder a $99 \%$ dos casos de SC. Uma vez diagnosticada a SC, é necessário estabelecer seu diagnóstico etiológico realizando-se exames laboratoriais mais específicos e exames radiológicos (BARBOSA et al., 2011; COSTENARO et al., 2012).

\section{Tireotropinoma}

Tireotropinomas representam menos de $1 \%$ dos AH (1:1.000.000). Afeta ambos os sexos de forma semelhante e a idade média de diagnóstico é em torno dos 41 anos. A maioria dos casos corresponde a macroadenomas (CARON, 2009a).

A apresentação clínica é similar à da Doença de Graves (hipertireoidismo primário), com algumas diferenças. O bócio, geralmente é difuso, mas pode ser multilobular. Exoftalmia é rara e, quando 
ocorre, é unilateral (BECK \& PERSANI., 2008).

A tireotoxicose apresenta vários sinais e sintomas, tais como: nervosismo, irritabilidade, suor excessivo, pele úmida, tremores, perda de peso, hipertensão, taquicardia, fraqueza muscular, insônia, bócio e alterações menstruais (BARBOSA et al., 2011; ALHOSEINI \& MOVAGHAR, 2012).

O diagnóstico correto é frequentemente feito em consequência dos sintomas neurológicos e da falha da terapia, visto que os pacientes são tratados inicialmente por engano, e por um longo período, para hipertireoidismo primário (DUARTE et al., 2009).

\section{Gonadotropinomas}

Os gonadotropinomas podem produzir apenas pequenas quantidades de FSH e LH e são classificados também como adenomas clinicamente não funcionantes (CURY et al., 2009).

\section{Somatotropinomas}

Os somatotropinomas correspondem a mais de $95 \%$ dos casos de acromegalia, e o diagnóstico dessa desordem debilitante é realizado por volta de 8 a 10 anos após o surgimento dos primeiros sintomas (BENSHLOMO \& MELMED, 2008). Os principais sintomas da acromegalia podem ser atribuídas ao processo de crescimento de mãos e pés, alargamento da região frontal, queixo proeminente, espaçamento entre os dentes e perda dentária, aumento do volume do tórax, nariz e dos lábios, espessamento da pele - que se torna oleosa e propensa à acne -; sudorese excessiva, especialmente nas mãos, alterações respiratórias, cardiovasculares, gastrintestinais, metabólico-endócrinas, músculo-esqueléticas, neurológicas e oftálmicas. Quando a doença surge na adolescência, pode haver o aumento exagerado da altura do paciente, levando ao quadro de gigantismo (ASA, 2008).

\section{ALTERAÇÃO NOS EXAMES LABORATORIAIS}

\section{Prolactinoma}

O diagnóstico de prolactinoma está baseado na presença de níveis de prolactina acima de $200 \mathrm{ng} / \mathrm{ml}$ em pacientes com lesão expansiva hipofisária maior do que $1,0 \mathrm{~cm}$ na ressonância magnética. A função hipofisária deve ser avaliada nos portadores de microprolactinomas, incluindo GH e Fator de Crescimento Semelhante à Insulina tipo 1 (IGF-1), para verificar a possibilidade de um tumor cossecretor (GLEZER \& BRONSTEIN, 2014). 


\section{Corticotropinoma}

Entre os exames confirmatórios do hipercortisolismo estão a ausência de supressão do cortisol após $1 \mathrm{mg}$ de dexametasona "overnight" - que é um antiinflamatório e imunossupressor dos corticosteroides -, o aumento da excreção do cortisol livre na urina de 24 h (no mínimo duas medições), a presença de um cortisol elevado à meia- noite, seja por cortisol salivar ao deitar (no mínimo duas medições) ou por cortisol sérico e ainda o cortisol sérico não suprimido após $2 \mathrm{mg} / \mathrm{dia}$ de dexametasona em 48h (LYNNETTE et al., 2008).

$\mathrm{Se}$ os resultados destes testes apresentarem valores de referência normal: masculino no período matutino 1-76 ng/l e feminino no período matutino 1-73 ng/l, a presença de Síndrome de Cushing é pouco provável. Contudo, é necessária a presença de, no mínimo, dois testes alterados para realização do diagnóstico sindrômico. Para a exclusão do diagnóstico da SC, é necessária a realização de, no mínimo, dois testes em momentos diferentes (LYNNETTE et al., 2008).

O diagnóstico etiológico de Doença de Cushing, nos pacientes, é estabelecido por meio de: ACTH sérico normal ou elevado; supressão do cortisol sérico ou urinário após a administração de $8 \mathrm{mg}$ de dexametasona “overnight” ou $2 \mathrm{mg} \mathrm{6/6h}$ por 48h; teste de estimulação com Hormônio Liberador de
Corticotrofina (CRH) com aumento de ACTH e cortisol

(ACTH-dependente)

(COSTENARO et al., 2012).

\section{Somatotropinoma}

$\mathrm{Na}$ acromegalia os níveis séricos de GH basal estão aumentados acima de $5 \mu \mathrm{g} / \mathrm{L}$. O IGF-1 comparados à idade e sexo estão aumentados. Apenas um único teste não confirma ou exclui o diagnóstico de acromegalia devido à secreção pulsátil de GH (BARBOSA et al., 2011).

O diagnóstico de acromegalia é confirmado pela ausência de supressão e/ou aumento paradoxal do GH acima de $2 \mu \mathrm{g} / \mathrm{L}$ após uma sobrecarga de glicose anidra $75 \mathrm{~g}$ (TOTG). O principal impedimento para sua realização é a presença de diabetes mellitus associado. (BARBOSA et al., 2011).

\section{Tireotropinoma}

Os tireotropinomas caracterizam-se pela presença de níveis séricos aumentados de T3 e/ou T4 associados a valores de TSH elevados ou normais, porém não adequados para os níveis dos hormônios periféricos. Ocorre também, ausência de resposta do TSH à administração de TRH (75-80\% dos casos), falta de supressão do TSH pelo T3 (em 80\%), níveis séricos elevados de subunidade alfa (64\% dos casos), a elevação do seu nível sérico e da sua relação molar com o TSH é 
altamente sugestiva de tireotropinoma. A hipersecreção do TSH pode ser isolada ou associada à outros hormônios hipofisários (BECK \& PENSANI, 2008; DUARTE et al., 2009; BARBOSA et al., 2011).

\section{ALTERAÇÕES NOS EXAMES RADIOLÓGICOS}

A tomografia computadorizada tem sido utilizada na prática médica desde a década de 70. O aparelho consiste em uma fonte de raios-X que é acionada ao mesmo tempo em que realiza um movimento circular ao redor do corpo do paciente, emitindo um feixe de raios-X em forma de leque. No lado oposto a essa fonte, está localizada uma série de detectores que transformam a radiação em um sinal elétrico que é convertido em imagem digital. Dessa forma, as imagens correspondem a secções ("fatias") do corpo. A intensidade (brilho) dos tecidos biológicos na imagem de TC reflete a absorção dos raios-X e pode ser medida em uma escala (unidades Hounsfield). Porém, ainda é limitada a capacidade da TC de diferenciar a substância branca e cinzenta do Sistema Nervoso Central (SNC), especialmente na região do cerebelo e núcleos da base (WERLANG et al., 2009).

Embora seja um exame radiológico mais facilmente obtido e de menor custo que a RM, a TC de sela túrcica e região parasselar somente oferece vantagem na melhor visualização da invasão de estruturas ósseas, tais como o assoalho selar (Figura 1) (VILAR, 2013).
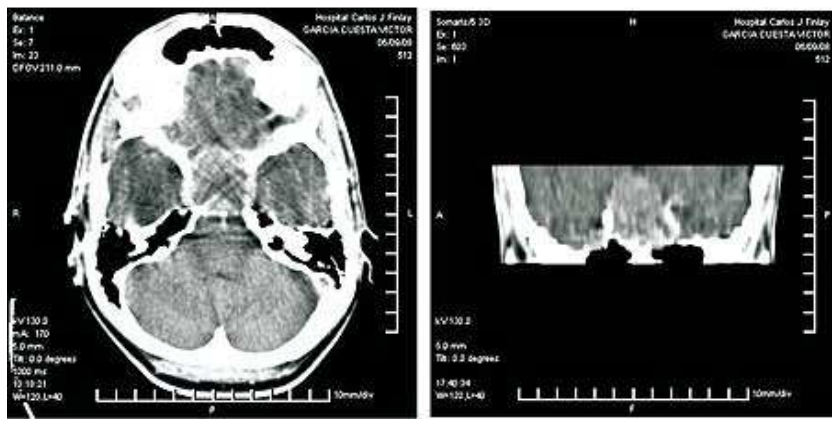

Figura 1. TC de sela túrcica corte axial (à esquerda) e coronal (à direita), mostrando macroadenoma hipofisário e diagnóstico anatomopatológico. Fonte: SÁNCHEZ \& ALFONSO, 2010.

A Ressonância Magnética é um fenômeno físico de troca de energia entre força periódica (ondas eletromagnéticas) e corpos em movimento. A condição para que ocorra o fenômeno da ressonância é que a frequência periódica das ondas eletromagnéticas seja igual à frequência de movimento dos corpos (HAGE \& IWASAKI, 2009).

O fenômeno da ressonância aplicada ao diagnóstico por imagem se baseia na troca de energia entre núcleos de átomos de hidrogênio com ondas eletromagnéticas provenientes de campos magnéticos oscilatórios. Para que este processo ocorra de forma controlada é necessário que os núcleos de hidrogênio estejam alinhados a um campo magnético externo. Quando forem aplicadas as ondas eletromagnéticas externas (ondas de radiofrequência), os spins de hidrogênio absorverão energia e mudarão de orientação em relação ao campo magnético. Quando o 
sinal de radiofrequência cessa, os spins de hidrogênio se alinham novamente na mesma direção do campo, liberando energia sob a forma de sinal de RM nuclear (captado por bobinas específicas ou bobinas receptoras) (HAGE \& IWASAKI, 2009; WERLANG et al., 2009).

A obtenção de imagens por ressonância a partir do hidrogênio se deve ao fato de este elemento estar amplamente distribuído nos tecidos biológicos e por suas características em responder a campos magnéticos externos como se fosse um pequeno íma (possui o maior momento magnético, o que aumenta a sensibilidade do exame para diferenciar tecidos biológicos normais e patológicos) (HAGE \& IWASAKI, 2009; MAZZOLA, 2009).

$\mathrm{O}$ aparelho de RM consiste em um túnel com cerca de 1,5 a 2,5 metros de comprimento e produz um ruído durante a emissão das ondas de radiofrequência e procedimento de localização do sinal. Esse ambiente é limitante para claustrófobos, contra-indicado para pacientes com marcapasso e "clips" de aneurismas, porém, há outras contra-indicações formais (MAZZOLA, 2009; WERLANG et al., 2009).

As imagens de RM têm maior capacidade de demonstrar diferentes estruturas no cérebro por apresentar técnicas altamente sensíveis para mínimas alterações na maioria das doenças do Sistema Nervoso Central. Essas técnicas incluem T1, T2 e T1 realçadas com contraste gadolínio (Figura 2) (MILANO, 2010).
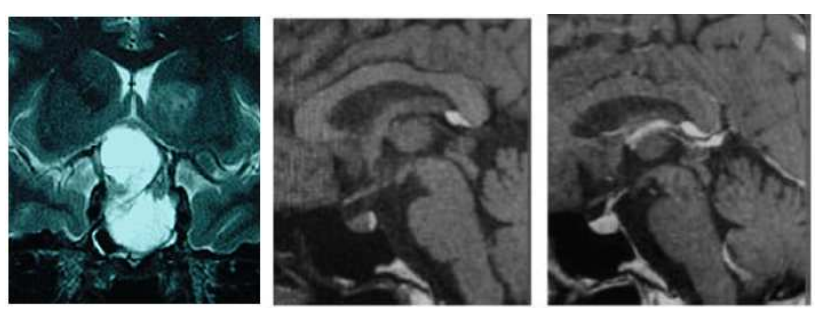

Figura 2. Imagens da região selar por RM. À esquerda coronal T2 com macroadenoma. Imagens sagitais T1, central e à direita, sem e com contraste da região selar normal. Fonte: REVOLLO \& MOLINA, 2012; anatpat.unicamp.br/rpgadenoma8.html.

Na RM, as imagens são avaliadas de forma a se obter o diagnóstico preciso da lesão, bem como análise de suas características morfológicas. Os adenomas apresentam sinal menor que o restante do parênquima em T1, sendo bem evidenciáveis em até 80 a $85 \%$ dos casos sem o uso do meio de contraste. O sinal em T2 é variável, dependendo de suas características internas. O uso do contraste gadolíneo pode auxiliar no diagnóstico de lesões pouco evidentes em T1 sem contraste, particularmente na Doença de Cushing.

O gadolíneo é um elemento químico metálico, branco prateado, maleável, da série dos Lantanídeos e de estrutura cristalina hexagonal, possui aspecto semelhante ao aço, com propriedades supercondutoras e é quimicamente muito ativo. Este contraste provoca o encurtamento do tempo de relaxação longitudinal, intensificando o sinal dos tecidos em sequências ponderadas em T1 (VILAR, 2013) (Figuras 3). 
Macroadenomas são geralmente bem evidentes na RM, porém apresentam sinal mais heterogêneo em T1 e T2. Calcificações são raras, e a administração do contraste endovenoso é essencial na caracterização da lesão, especialmente em sequências “dinâmicas" nas quais se observam lesões que realçam em função do tempo após a administração do contraste (VILAR, 2013). Portanto, a RM é de grande valor na visualização do quiasma óptico e do trato óptico e em avaliar o grau de expansão tumoral (WERLANG et al., 2009).

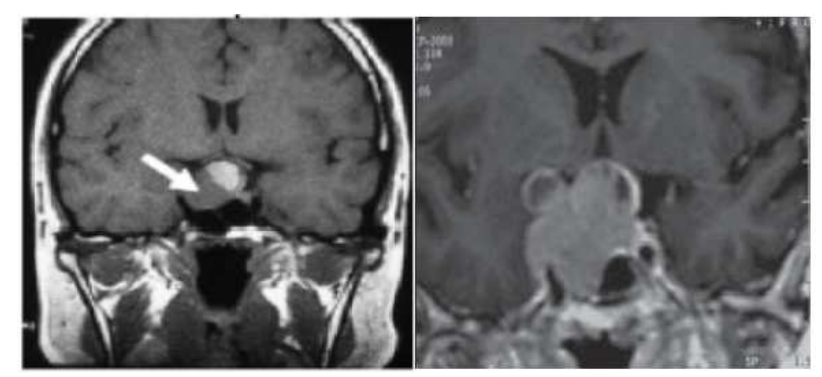

Figura 3. RM da sela túrcica corte coronal, fase T1, posterior à administração de gadolíneo. Na figura à esquerda observa-se hiperintensidade esquerda intraselar. Na figura à direita destaca-se a invasão do seio cavernoso direito que se evidencia por lesão da carótida. Fonte: ROJAS et al., 2008; REVOLLO \& MOLINA, 2012.

\section{DISCUSSÃO}

Os Adenomas hipofisários são a patologia mais comum entre as massas selares (90\% dos casos). As demais etiologias incluem craniofaringioma, meningioma, pituicitomas, glioma, cordoma, tumores metastáticos, cisto da bolsa de Rathke, hiperplasia hipofisária fisiológica e patológica, dentre outras. A distinção entre essas lesões não é fácil, visto que muitas delas podem ser semelhantes, do ponto de vista clínico, endócrino e radiológico. A avaliação hormonal é indicada em praticamente todos os casos de lesões da região selar. Em muitos casos só será feito o diagnóstico etiológico através do exame anatomopatológico da lesão (imunohistoquímica) (GLEZER et al., 2008; JANE, 2008).

O prolactinoma constitui a principal causa de hiperprolactinemia, representando a maioria dos casos de adenomas hipofisários (VILAR et al., 2008; MIYAI et al., 1986 apud GLEZER \& BRONSTEIN, 2014). Entretanto, a hiperprolactinemia pode ser resultante de diversas causas fisiológicas, farmacológicas e patológicas, tais como: gravidez e amamentação, antipsicóticos e antidepressivos, $\mathrm{AH}$ mistos, comprometimento da haste hipofisária, macroprolactinemia, hipotireoidismo primário grave e de longa duração (leva a uma hiperplasia hipofisária intensa), dentre outros. Portanto, confirmada a hiperprolactinemia, sua etiologia deve ser investigada adequadamente e vários fatores devem ser considerados, tais como: história clínica (uso de medicamentos, descartar gravidez, doenças que elevem a PRL), exame físico (presença de lesões irritativas ou traumáticas na parede torácica) e exames de imagem da sela túrcica (DEMSSIE \& DAVIS, 2008).

Para se evitar o diagnóstico errôneo os exames de imagem geralmente devem ser 
realizados somente após a exclusão de hiperprolactinemia de causa fisiológica, farmacológica ou decorrente de doenças sistêmicas, como hipotireoidismo primário, cirrose ou insuficiência renal (PRABHAKAR \& DAVIS, 2008). O correto diagnóstico dos prolactinomas tem importância fundamental para um tratamento adequado. O principal diagnóstico diferencial é com os chamados psedoprolactinomas, que determinam a elevação dos níveis de PRL por compressão da haste hipofisária e usualmente requerem a cirurgia como terapia. Em contraste, os prolactinomas são prioritariamente tratados com os agonistas dopaminérgicos (MANCINI, et al, 2008; DELEMER, 2009).

$\mathrm{Na}$ acromegalia, apesar de as manifestações clínicas serem bastante características, com evolução insidiosa, elas são motivo de consulta em somente cerca de $10 \%$ dos pacientes. O diagnóstico frequentemente é realizado em torno de 8 a 10 anos após o aparecimento dos primeiros sintomas. Tal fato é bastante preocupante, uma vez que o diagnóstico precoce resulta em um tratamento que pode evitar ou minimizar o surgimento de complicações cardiovasculares, respiratórias e neoplásicas da doença. Níveis elevados do GH sérico podem, também, ser observados em indivíduos com produção ou ação deficiente do IGF-I ou de suas proteínas carreadoras por mecanismo de feedback: diabetes, cirrose, anorexia nervosa, doenças agudas, AIDS e desnutrição (MELMED \&
KLEINBERG, 2008).

A Doença de Cushing resulta de um corticotropinoma e deve ser diferenciada de outras causas de síndrome de Cushing, incluindo causas supra-renais, produção ectópica de ACTH e CRH e causas fisiológicas que resultam em hipercortisolimo. As manifestações clínicas são variáveis do aumento da produção de cortisol principalmente nos casos leves ou no início da doença, o que dificulta o diagnóstico (MELMED \& JAMESON, 2013).

Os pacientes podem apresentar sintomas clínicos que são causados pelo excesso de cortisol e manifestações múltiplas que são frequentes na população como: depressão, irregularidade menstrual, obesidade e diabetes. Portanto, a suspeita clínica é importante, visto que estabelece a triagem antes da realização dos exames laboratoriais (NEWELL-PRICE, 2010).

Os adenomas hipofisários clinicamente não funcionantes são a causa mais frequente de macroadenomas. Como não possuem clínica de hipersecreção hormonal, são diagnosticados incidentalmente (cerca de $15 \%$ dos casos) ou quando já apresentam uma grande extensão selar com sintomas compressivos e hipopituitarismo. A avaliação laboratorial é importante para detecção de hipersecreção hormonal, essencial no diagnóstico diferencial com adenomas funcionantes. Os exames solicitados são: dosagem de PRL, GH, IGF-1, ACTH, 
cortisol, LH, FSH, TSH, tiroxina livre, testosterona e estradiol. A RM realizada sem e com contraste, e com imagens em T1 e T2, é muito importante no diagnóstico diferencial de outros tumores da região, como craniofaringioma, abscesso, cisto da bolsas de Rathke, lesão vascular com aneurisma, dentre outros. Embora com menor poder de resolução, a tomografia computadorizada poderá revelar maior clareza de calcificações e integridade óssea (MOLITCH, 2008; CARON, 2009b).

\section{CONCLUSÃO}

Apesar de ser a alteração hipofisária mais comum, o adenoma hipofisário é uma doença de difícil diagnóstico, pelo seu crescimento lento, com manifestação clínica tardia e diversificada. Os exames laboratoriais usados na dosagem dos hormônios hipofisários juntamente com a clínica do paciente e os exames de imagem, tomografia computadorizada e ressonância magnética, são essenciais para a elucidação do diagnóstico dos adenomas hipofisários. Esse último exame tornou-se a mais promissora técnica de diagnóstico radiológico, complementando com alta sensibilidade os achados clínicolaboratoriais de pacientes com adenoma. Isso se deve à excelente resolução e contraste que as imagens de ressonância exibem. Por isso, a RM da sela túrcica tem sido o exame mais indicado para o diagnóstico dessa patologia.

\section{REFERÊNCIAS BIBLIOGRÁFICAS}

ALHOSEINI, M.S.; MOVAGHAR, V.R. Pituitary adenomas: A Review. Journ of Inj and Viol, v.4, p. 56, 2012.

ALVES, M. et al. Adenoma atípico da hipófise ou corticotrofinoma maligno? Acta Med Port, v. 24, p. 661-666, 2011.

ASA, S. L. Practical Pituitary Pathology What Does the Pathologist Need to Know? Arch Pathol Lab Med, v. 132, p. 1231-1240, 2008.

BARBOSA, E.R. et al. Controle hormonal dos adenomas hipofisários pela cirurgia transesfenoidal: evolução dos resultados nos primeiros cinco anos de experiência. Arq Bras Endocrinol Metab, v. 55, p. 16-28, 2011.

BEN-SHLOMO, A.; MELMED, S. Acromegaly. Endocrinol Metab Clin North Am, v. 37, p. 101-122, 2008.

BECK, P.P.; PERSANI, L. Thyrotropinnomas. Endocrinol Metc Clin North Am, v.37, p. 123-134, 2008.

CARON, P. Clinically non functioning pituitary adenomas and gonadotroph-cell adenomas, Presse Med, v.38, p. 103-106, 2009.

CARON, P. Thyrotropin-secreting pituitary adenomas. Presse Med, v. 38, p. 107-111, 2009.

COSTENARO, F. et al. Avaliação do eixo hipotálamo-hipófise adrenal no diagnóstico e na remissão da doença de Cushing. Arq Bras Endocrinol Metab, v.56, p. 159-167, 2012.

CURY, M.L.C.A.R. et al. Non-functioning pituitary adenomas: clinical feature, laboratorial and imaging assessment, 
therapeutic management and outcome. Arq Bras Endocrinol Metab, v. 53, p. 31-39, 2009.

DELEMER, B. Prolactinomas: diagnosis and treatment. Presse Med, v. 38, p. 117-124, 2009.

DEMSSIE Y.N.; DAVIS J.R.

Hyperprolactinemia. Clin Med, v. 8, p.216219, 2008.

DUARTE, F.H.G. et al. Tumores hipofisários secretores de TSH: relato de dois casose revisão da literatura. Arq Bras Endocrinol Metab, v. 53, p. 1157 -1166, 2009.

GLEZER, A.; BRONSTEIN, M.D.

Prolactinoma. Arq Bras Endocrinol Metab, v. 58, p. 118-123, 2014.

GLEZER, A. et al. Rare sellar lesions. Endocrinol Metab Clin North Am, v. 37, p. 195-211, 2008.

GOMES, M. Tumores da hipófise: contribuição do estudo clínico e molecular para o conhecimento da patogenia e comportamento biológico dos tumores clinicamente não funcionantes. Tese de Doutorado. Faculdade de Medicina. Universidade de Coimbra, 2011.

HAGE, M.C.F.N.S.; IWASAKI, M. Imagem por ressonância magnética: princípios básicos. Ciência Rural, Santa Maria, v. 39, p.1287-1295, 2009.

JANE, J.A. Manageement of pediatric sellar tumors. Pediatr Endocrinol Rev, v. 5, p.720726, 2008.

LAKE, M.G. et al. Pituitary Adenomas: An Overview. American Family Physician, v. 88, p. 319-327, 2013.

LYNNETTE K. N. et al. The Diagnosis of Cushing's Syndrome: An Endocrine Society Clinical Practice Guideline. J Clin Endocrinol Metab, v. 93, p. 1526-1540, 2008.

MANCINI, T. et al. Hyperprolactinemia and prolactinomas. Endocrinol Metab Clin North Am, v. 37, p. 67-99, 2008.
MAZZOLA, A.A. Ressonância magnética: princípios de formação da imagem e aplicações em imagem funcional. Rev Bras de Fís Med, v. 3, p.117-129, 2009.

MELMED, S.; JAMESON, J.L Distúrbios da adenohipófise e do hipotálamo. In:

BRAUNWALD, E. et al. MEDICINA

INTERNA DE HARRISON. $18^{a}$ edição. New York: Mc Graw-Hill, 2013. cap. 339, p. 28762901.

MELMED, S.; KLEINBERG, D. Anterior Pituitary. In: KRONENBERG, H.M. et al. Williams Textbook of Endocrinology. 11 ${ }^{\mathrm{a}}$ edição. Philadelphia, 2008. p. 155-262.

MILANO, J. B. Estudo das alterações em exame de ressonância magnética de pacientes em pós-operatório imediato de ressecção de tumores hipofisários por via transesfenoidal. Tese de Douturado. Faculdade de Medicina da Universidade de São Paulo, p. 22, 2010.

MOLITCH, M.E. Nonfunctioning pituitary tumors and pituitary incidentalomas. Endocrinol Metb Clin North Am, v. 37, p.151-171, 2008.

MOLITCH, M.E. Hipófise Anterior. In: GOLDMAN, L.; SCHAFER, A.I. Cecil Tratado de Medicina Interna. 24a edição. Rio de Janeiro: Elsevier, 2014. cap. 231, p. 16431658.

NEWELL-PRICE, J. Etiologies of Cushing's Syndrome. In: BRONSTEIN, M.D. Cushing's Syndrome: Pathophysiology, Diagnosis and Treatment. $1^{\text {a }}$ Edição. New Jersey: Humana Press, 2010. cap. 2, p.21-30.

PLATA, M.L.A.O, et al. Pituitary Adenomas - Clinico-Pathological, Immunohistochemical and Ultrastructural Study. National Institute of Neurology and Neurosurgery, México City, 2012, p. 49-66. Disponível em: $<$ http://cdn.intechopen.com/pdfswm/28177.pdf> Acesso em: agosto, 2014.

PRABHAKAR, V.K.; DAVIS, J.R.

Hyperprolactinemia. Best Pract Res Clin 
Obstet Gynaecol, v. 22, p. 341-353, 2008.

REVOLLO, M.C.; MOLINA, F.C.

Resonancia Magnética En Adenomas de

Hipófisis. Rev Med La Paz, v.18, 2012.

Disponível em:

$<$ http://www.scielo.org.bo/scielo.php?pid=S1

726-

$89582012000200005 \&$ script=sci_arttext $>$

Acesso em: setembro, 2014.

ROJAS, Z.D.; et al. Manejo de los adenomas hipofisiarios. Rev Chil Neuro-Psiquiat, v. 46, p.140-147, 2008.

SÁNCHEZ, S.G.; ALFONSO, L.S.

Coincidencia de la tomografía

computadorizada monocorte con el examen

anatomopatológico posquirúrgico en el

diagnóstico de los tumores intracraneales

primários. Rev Cub Med Milt, v.39, p.125-

133, 2010.

VELOZA, A.; PRAZERES, S. Prolactina e o

Laboratório. Acta Med Port, v. 24, p. 1029-

1034, 2011.

VILAR, L. Parte 1 Neuroendocrinologia. In:

Endocrinologia clínica. $5^{\text {a }}$ edição. São Paulo:

Guanabara Koogan, 2013. cap. 1-11, p. 3-131.

VILAR, L. et al. Diagnosis and management of hyperprolactinemia: results of a Brazilian multicenter study with 1234 patients. Jornal

Endocrinol Invest, v.31, p. 436-444, 2008.

WERLANG, et al. Manual do residente de radiologia. $2^{\text {a }}$ Edição. Rio de Janeiro:

Guanabara Koogan, 2009, cap. 1, 2, 6, 8, p. 01-128. 